\title{
Citation Auctions as a Method to Improve Selection of Scientific Papers
}

\author{
Josep Lluis de la Rosa \\ University of Girona, Girona, Catalonia, \\ EU peplluis@eia.udg.es
}

\author{
Boleslaw K. Szymanski \\ Rensselaer Polytechnic Institute, Troy, NY, \\ USA szymansk@cs.rpi.edu
}

\begin{abstract}
This paper describes the basis of citation auctions as a new approach to selecting scientific papers for publication. Our main idea is to use an auction for selecting papers for publication through - differently from the state of the art - bids that consist of the number of citations that a scientist expects to receive if the paper is published. Hence, a citation auction is the selection process itself, and no reviewers are involved. The benefits of the proposed approach are two-fold. First, the cost of refereeing will be either totally eliminated or significantly reduced, because the process of citation auction does not need prior understanding of the paper's content to judge the quality of its contribution. Additionally, the method will not prejudge the content of the paper, so it will increase the openness of publications to new ideas. Second, scientists will be much more committed to the quality of their papers, paying close attention to distributing and explaining their papers in detail to maximize the number of citations that the paper receives. Sample analyses of the number of citations collected in papers published in years 1999-2004 for one journal, and in years 2003-2005 for a series of conferences (in a totally different discipline), via Google scholar, are provided. Finally, a simple simulation of an auction is given to outline the behaviour of the citation auction approach.
\end{abstract}

\section{Introduction}

This work is the result of our insight and reflection on the refereeing process under which the scientific community has been suffering for many decades. The peer review (known as refereeing in some academic fields) is a process of subjecting an author's scientific work or ideas to the scrutiny of one or more others who are experts in the field. It is used primarily by publishers and funding agencies to select and to screen submissions of manuscripts and in awarding of funding for research. The process aims at getting authors to meet the standards of their discipline and thus achieve scientific objectivity. Publications and awards that have not undergone peer review are likely to be regarded with suspicion by scholars and professionals in many fields. However, the peer review, though universally used, is slow, time and resource consuming, and obstructs the emergence of new ideas by subjecting them to the control of prevailing dogmas or cliques. Moreover, highly prestigious qualified reviewers are a bottleneck of the peer review process, as there are not many of them, so they are overloaded or simply missing. These drawbacks of peer review create a big problem for journals and conferences. We have observed that it is not unusual for a general chair of a conference to desperately seek reviewers very close to the deadline of announcing acceptance/rejection decisions for some fraction of submissions. Quality of such last moment peer reviews of course will remain questionable.

Our proposal is to replace the review process by an auction based approach: the better the submitted work, the more the author may bid to have it appear in some conferences or journals. If the assessment of quality represented by the bid is right, the author will be rewarded in some new sort of scientific currency, otherwise the author will suffer loses of this currency. In this paper, we will argue that citations can be an appropriate world-wide accepted scientific currency. We will also conjecture that citation auction will encourage better self-control of the submission quality by the scientists, will inspire them to prepare more exciting talks for accepted papers and invite the discussion of their results at congresses and conferences and among colleagues or students and their supervisors. Ultimately, this paper discusses how a citation auction can motivate and influence scientists. We believe that this idea may bring together researchers, designers, and developers interested in computer systems capable of changing human attitudes and behavior in positive ways.

We remark that our approach is not about reviewers bidding to review papers [14] that are still selected after the reviewing process, but a citation auction is the selection process itself, and no a priori reviewers are necessary.

Section 2 presents the drawbacks of peer review process and Section 3 shows those of the current impact factors. Section 4 introduces the proposal of a new citation based currency and the outline of citation auction approach. Section 5 shows some 
tools for looking up citations. Section 6 describes hypothetical cases of a citation auction and provides its analysis. Finally, preliminary short discussion and conclusions are included in Section 7.

\section{Drawbacks of the Peer Review}

While some believe passing the peer review process is a certificate of validity, others often hold a far more sceptical view.

One of the most common complaints about the peer review process is that it is slow, and that it typically takes several months, or even several years in some fields, for a submitted paper to appear in print. Such a delay in a fast-growing field is devastating for propagation of ideas and needs a solution. For example, much of the communication about new results in some fields such as astronomy no longer takes place through peer reviewed papers, but rather through preprints submitted onto electronic servers such as arXiv.org.

In addition, some sociologists of science argue that peer review makes the ability to publish susceptible to control by elites and to personal jealousy. The peer review process may suppress dissent against "mainstream" theories. Reviewers tend to be especially critical of conclusions that contradict their own views, and happily accept those that accord with them. At the same time, elite scientists are more likely than less established ones to be sought out as referees, particularly by high-prestige journals or publishers. As a result, it has been argued, ideas that harmonize with the elite's views are more likely to be seen in print and to appear in premier journals than are iconoclastic or revolutionary ones and therefore the whole process obstructs and delays the emergence of new ideas and scientific revolutions.

However, others have pointed out that there are many scientific journals in which one can publish, making control of information difficult. In addition, the decision-making process of peer review, in which each referee gives his opinions separately and without consultation with the other members, is intended to mitigate some of these problems.

Moreover, the peer review tends to accept those weaker papers which show a mix of prestigious coauthors together with unknown authors. This is because the typical referee's behaviour of trusting those papers with prestigious authors even if the reviewer cannot fully understand a given paper contributions, believing "this must be true, must be good, surely they will correct any potential drawbacks of this paper in the conference". This may not systematically be bad, but there are more elegant ways to give opportunities to new authors. Moreover, the enormous responsibility of referees might be reduced if some sort of penalty for the authors existed when the contributions were not as good as required by the standards of a publication. The fact is that for the sake of improving the quality of science, the trust on important co-authors should be reduced in a public but not in aggressive way. However, the blind peer review process, that could be considered such an approach, is in fact yet another imperfect solution.

New solutions to redress many of the problems of traditional peer review are shown in the recently launched (2006) online journal Philica, which, unlike a traditional journal, publishes immediately all articles submitted to Philica and the review process takes place afterwards. Reviews are still anonymous, but instead of reviewers being chosen by an editor, any researcher who wishes to review an article can do so. Reviews are displayed at the end of each paper, and so are used to give the reader criticism or guidance about the work, rather than to decide whether it is published or not. This means that reviewers cannot suppress ideas if they disagree with them. We will pick this idea of a citation auction in the following sections.

Some authors (e.g., Mizzaro [10]) suggest scoring papers and authors, letting the readers to act directly as referees, and receiving feedback on the readers for achieving good quality judgments, so that Good readers have good reputation. We will take further this idea and adapt it for a citation auction.

Another very interesting approach for overcoming the drawbacks of peer-review has been used by the European commission for a specific research program called Future and Emerging Technologies (FET), where proposals are not peer-refereed but voted by researchers being considered for evaluation.

\section{Citations: pros and cons}

The peer review is not the only problem of the scientific community. Another problem is the overuse of the analysis of citations - examining what scientists publish - for the purpose of assessing their productivity, impact, or prestige which has become a cottage industry in higher education. However, it is an endeavour that also begs more scrutiny and scepticism. This approach has been taken to the extremes both for the assessment of individuals and as a measure of the productivity and influence of entire universities or even academic systems. Pioneered in the $1950 \mathrm{~s}$ in the United States, bibliometrics was invented as a tool for tracing research ideas, the progress of science, and the impact of scientific work. Developed for the hard sciences, it was expanded to the social sciences and humanities as well. 
According to [1] the citation system was invented mainly to understand how scientific discoveries and innovations are communicated and how research functions. It was not, initially, seen as a tool for the evaluation of individual scientists or entire universities or academic systems. Hence, the citation system is useful for tracking how scientific ideas in certain disciplines are circulated among researchers at top universities in the industrialized countries, as well as how ideas and individual scientists use and communicate research findings. The misuse of citation analysis distorts the original reasons for creating bibliometric systems. Evaluators and rankers need to go back to the drawing board to think about a reliable system that can accurately measure the scientific and scholarly work of individuals and institutions. The unwieldy and inappropriate use of citation analysis and bibliometrics for evaluation and ranking does not serve higher education well - and it entrenches existing inequalities.

More recently there is a new index, the index $h$, defined as the number of papers with numbers of citations at least $h$, proposed for characterizing the scientific output of a researcher [16]. For example, an author with index $\mathrm{h}=20$ must have 20 papers with at least 20 citations each. This index can easily be found ordering papers by "times cited" in the Thomson ISI Web of Science or Google Scholar and it gives an idea about the ratio of productivity of publications in terms of citations, and not only about the mass of citations. This concept will also be combined with the approach of [13] in the citations auction proposal.

Summarizing, the drawbacks of the citation based analysis are overemphasis of certain languages, bias towards hard sciences, and focus on traditional scholarly journals. A step forward, though not enough, is the $h$ index that avoids some of the disadvantages of several criteria based only on citations [13]. We propose more radical solution and argue that all the drawbacks may be partially solved and even lead to new benefits, by introducing some principles from economics as well as electronic commerce, in the form of auctions.

\section{Citations as a new world wide accepted scientific currency, and auctions}

At the same time, the citation system serves well the purposes for which it was invented. This paper expands from [11], the proposal of yet another proper use of this system to create an alternative to the existing a priori peer reviews. Today, conferences and workshops often suffer from low participation and little discussion of the presented papers. Authors focus mostly on getting their papers to the conference rather than focusing on the wider dissemination of their results. This is the impact problem. Likewise, the organizers extend extraordinary effort trying to select the best paper for the conferences and thus creating a heavy refereeing workload. This is the refereeing problem. To avoid these pitfalls, we propose to combine predictive value of citation and intimate knowledge of the papers by their authors into an auction system that promises to solve these problems. Citations, as a scarce resource, can be considered as currency [16], useful for auctions.

From the auction point of view, the current situation can be characterized as follows. Nowadays, a scientist wants to publish his scientific results in conferences and journals (CJs) to gain citations and reputation. Citations can be converted into a world wide accepted currency so that scientists that accumulate it can use them to trade for scientific purpose in citation auctions. Since there are too many scientists trying to publish in the same highly ranked and read CJs, then each of those CJs can select only a small number of submitted papers, hopefully those which will generate the maximum number of citations. Thus, in the citation auction approach, the CJs will activate an auction to select those papers to which the most citations are conjectured by its authors, or more precisely, according to the scientists bid. Such a bid represents a prediction given by an author about the number of citations that a paper will receive. To make this bid trustworthy, we consider the number of citations that this author has received from his previously published papers as his citations wallet (CW), i.e. the amount of "cash" that the author possesses. Therefore, authors are limited by their CWs in entering their bids, and they must speculate how many citations they will receive as a pay-back over time after the successful communication of their papers. As a result, every auction winning paper will withdraw the number of citations from its authors' $\mathrm{CW}$. Thus, what does the $\mathrm{CW}$ contain: one conference citations, a group of conferences citations, all papers? Our suggestion is either a group of conferences or all of the author's papers. This enables an author to collect $\mathrm{CW}$ in weaker conferences to get into a big one or into a journal. Consequently a rational author will bid with the highest number of citations they think that the paper will bring but to the limit of the wallet "cash" (i.e., past performance). Authors may lose their "cash" if they paid more citations in the winning bid than the number of citations that the paper would generate in the future, and vice versa, they may win more cash if the published papers generate more citations than those invested in an auction. The ultimate goal of an author will be to keep their individual $\mathrm{CW}$ up. For new scientists or late bloomers, a small fraction of papers for each conference can be accepted on the basis of traditional review process, however, only very outstanding papers should be accepted that way, 
avoiding the problem of the boundary quality papers that are the most difficult and most time consuming to evaluate currently.

Another approach for new scientists or late bloomers is to exploit a new feature of the citation auction, and this is to introduce the "citation loans" which could be enabled if another researcher (possibly the supervisor, advisor, boss, or good friend) is willing to lend part of his or her citations. In the case of a supervisor, this is clear, they are working together and there he is lending part of his prestige, that is his $\mathrm{CW}$, to his $\mathrm{PhD}$ student, as a type of investment, so that in the middle or long future his student will give him the citations back, eventually with extra citations in a new type of interest rate that the supervisor-student couple will agree on in advance or during the progress of the research, and that could be reflected in a contract-like agreement. Moreover, globally speaking, there is no fear that this approach would help further 'bad students with famous supervisors' than 'good students with unknown supervisors' because, the donors system based on the arbitrary but rational criteria of one scientist is more efficient than bureaucratic systems based on collectives of scientists that reached a consensus of what is good or bad.

The benefits of the proposed approach are two fold. First, costs of refereeing are reduced because the process of paper selection via a citation auction does not need a prior understanding of the paper content to evaluate the quality of its contributions. Second, authors will be much more committed to the quality of their papers, and will focus much more than today on wide dissemination and detailed explanations of their papers to maximize the number of citations. As a conclusion, this novel approach emphasizes the scientific collaborative work and active promotion of ideas, while reducing the expensive costs of current methods of refereeing and avoiding other possible faults like cliques and dogmas.

Now, let us have a look at what auctions are and how they can be applied to the evaluation of science and its impact. Let us start from the origin of auction in marketing. Traditional auctioneers consider advertising principles and "the psychology of selling" as key to auctioneering success. The growing popularity of Internet auctions is driving new product-market and pricing models [4] [5], revised channel roles, and new market research methods [6]. Let us set the stage with a brief description of the four major auction mechanisms, outline key concepts and results from the economic analysis of auctions, and summarize the key findings in empirical tests of auction theory.
We briefly describe the commonly discussed auction mechanisms, assuming that a single object is for sale and that a seller and several bidders operate without agents [7].

In an ascending-bid auction, the object's price is raised until only a single bidder remains. This winning bidder pays a price equal to his last bid (usually a small amount above the second highest bid). The auction is "open", i.e., the participants know the current best bid.

In a descending-bid ("Dutch") auction, the auctioneer starts with a high initial price and progressively lowers it. The prevailing price is posted and known to all participants. The first bidder to indicate a willingness to take the object at the prevailing price is the winner.

In contrast to an open auction, participants in a sealed bid auction submit their bids without seeing others' bids. In a first-price sealed bid (FPSB) auction, the highest bidder wins and pays his bid price. In a second-price sealed bid (SPSB) auction, the highest bidder also wins, but pays a price equal to the second highest bid.

Auction mechanisms are usually analyzed as noncooperative, incomplete (both symmetric and asymmetric) information games among competing bidders. The solution is based on the Bayesian Nash equilibrium in which bidders maximize their own expected payoffs from this single auction, conditional on their rivals' strategies and their beliefs about the rivals' information [8]. The baseline analysis assumes a single object auction and a set of $n$ symmetric and risk neutral bidders.

The questions are: will an auction be useful for scientific refereeing? Will the citation auction converge? Auction models differ in their assumptions about the bidders' information sets. In private value models, each bidder is assumed to know his own valuation of the object, but not others' valuations. In the case of citation auction, the object is the "paper" or synonymously the "contribution". In the baseline case, the valuations are assumed to be independent but drawn from a commonly known continuous distribution. Bidders' valuations vary, but are assumed to be unaffected by others' valuations. In contrast, with common value models, the object has the same common or true value for all bidders. However, bidders vary in their private signals (estimates) of the common value, with the signals assumed to be independent, drawn from the same continuous distribution. Bidders are uncertain of the object's worth and are influenced by information about others' signals revealed during the auction. In the case considered in this paper, bidders are the scientists who plan to publish their papers or contributions in a CJ, and the private value is the 
expected amount of citations that the paper will receive in the future, which will be measured by any of the worldwide accepted scientific citation systems. Since every scientist trusts his own work, he gives it a private value and tries to imagine what the common value of the other researchers will be. If papers are all made accessible in advanced (as is done in Philica journal) then all auctioneers may create a common value of all contributions.

This paper conjectures that the FSPB auction is the best approach for the citation auction. This is because in FSPB auction, the bids are not publicly shown but the contributions may be publicly available. This will let the scientific auctioneers have an internal estimation of IPV and an approximation to CV. However, the citation auction convergence does not necessarily relate with the real (final) value of the paper measured by the amount of citations through time over expectancies. The final amount of citations will depend not only on the quality of the paper (as stated in section 1) but on the author's extra effort invested in the dissemination of the paper not only in the CJs but in all events, seminars and talks (as [17] states) during the maturity life cycle of the paper. And what is claimed in this paper is that the higher the quality of the paper, the more people will cite it and those citations will exceed the expectations of the authors.

Finally, it is out of the scope of this paper, but such diffusion and promotion mechanism requires knowledge of deeper insight of the auction model. Hence, the new economic model derived from the citation auction will be needed to foresee its impact on increased quality of research, reduced peer review costs and time, etc.

\section{Tools for looking up citations}

There are several tools for tracing the citations of every work, such as the Scholar Google (see http://scholar.google.es/), the science indicators from ISI Web of Knowledge published by Thomson ( see http://isiknowledge.com), the computer science literature digital library ( see website http://citationseer.ist.psu.edu), the Elsevier scopus ( see http://www.scopus.com) and others.

As an example, to look up the citations of a scientist, in this case the search of the first author of this paper in years 1999-2004 focusing on Lecture Notes on Computer Science and Lecture Notes on Artificial Intelligence. For the sake of simplicity and easy replication of our results, we use Scholar Google, and the following table shows the amount of citations per year. Another ranking widely used in the scientific community is the ratio of citations to the number of publications, as described in [2] or [15]. So this researcher accumulated 8 citations in 6 publications, that gives him a ratio of $8 / 6=1.3$ citations per publication. The international average number of citations per publication in the fields of computer science and artificial intelligence is about 1.5 according to the International Science Indicators. Index $h=2$ is quite typical for a researcher in this field.

\begin{tabular}{|l|l|l|l|l|l|l|}
\hline Paper & 1999 & 2000 & 2001 & 2002 & 2003 & 2004 \\
\hline$\# 1$ & & 3 & & & & \\
\hline$\# \mathbf{2}$ & & 0 & & & & \\
\hline$\# 3$ & & 0 & & & & \\
\hline$\# \mathbf{4}$ & & & & 4 & & \\
\hline \#5 & & & & 0 & & \\
\hline \#6 & & & & & 1 & \\
\hline
\end{tabular}

\section{Hypothetical cases and their analyses}

\begin{tabular}{|l|l|l|l|}
\hline Period & $\begin{array}{l}\text { Total papers } \\
\text { cited }\end{array}$ & $\begin{array}{l}\text { Citations } \\
\text { per paper }\end{array}$ & $\begin{array}{l}\text { Citations per } \\
\text { author }\end{array}$ \\
\hline 2003 & 20 & 6.05 & 1.95 \\
\hline 2004 & 22 & 1.73 & 0.54 \\
\hline 2005 & 30 & 0.43 & 0.13 \\
\hline $2003-05$ & 73 & 2.38 & 0.75 \\
\hline
\end{tabular}

Table 1. Citations to papers published in PADS in 2003-2005

Let us consider papers accepted to the Workshop for Parallel and Distributed Simulation (PADS) in 2003-2005. There were 72 papers that generated 172 citations, so about 2.4 citations per paper. However, cumulatively, these papers had 230 authors, or 3.2 authors per paper. Hence, the individual citation ratio of each author of a paper was just 0.75. As expected, the number of cited papers was smaller for the later years, as not all citations were created yet for papers from 2004 and especially from 2005; these numbers are given in Table 1 . Note that each year a similar number of papers is published so the non-cited papers are not included in the analysis.

We selected four authors that had repeated papers published in PADS in the relevant period, denoted A1, A2, A3, A4, respectively. Their citations results are shown in Table 2.

\begin{tabular}{|l|l|l|l|}
\hline Author & $\begin{array}{l}\text { Total papers } \\
\text { cited }\end{array}$ & $\begin{array}{l}\text { Citations } \\
\text { per paper }\end{array}$ & $\begin{array}{l}\text { Citations } \\
\text { per author }\end{array}$ \\
\hline A1 & 9 & 5.11 & 1.53 \\
\hline A2 & 5 & 0.4 & 0.4 \\
\hline A3 & 2 & 5 & 1.6 \\
\hline A4 & 2 & 9 & 3.44 \\
\hline
\end{tabular}

Table 2. Citations to papers published in PADS in 2003-2005

Clearly, in terms of total number of citations, the most visible researchers are A1 (46), then A4, A3, 
and A2. In terms of number of citations per paper or per author, the authors A1 and A4 switch places. Since the average number of citations per author for the whole conference is 0.75 , only authors A4, A1 and A2 should be able to publish. If the number of citations per paper is considered, then also author A2 would not meet the criteria.

This brief analysis underlines again several problems that need a careful solution. First, if each author is credited with all citations to the paper, we will have an inflation of wallets, as the paper with five authors and five citations, bid with 5 citations will contribute 25 citations total to all authors' wallets, but the payment will be just 5 credits from the wallet of one author. Hence, one can think of dividing citations obtained by a paper equally to co-authors, but a more impartial decision is to assign the total of citations accordingly to a "citations contract" among authors submitting the paper in the citation auction. This contract will consist of a percentage of participation in authoring the paper. In case there is no "citations contract", the total amount will be assigned to the first author who will then decide how to share the citations among co-authors. However, not to discourage collaborations, we also allow all authors of the paper to contribute their credits to the joint bid. This will solve the inflation problem. Often coauthors are students, so they can build their wallets for a future independent career while working with their advisor on their theses.

The second inflation problem arises from selfreferences. If an author has a $\mathrm{CW}$ of 20 citations, this author can safely bid all 20 citations on the new paper if there are 20 citations to the author's work in the paper! After publishing the paper, the author will automatically receive the credit for the citations, immediately rebuilding the wallet. To avoid this effect, all self-citations by any author would not be added to the author's wallet.

The third problem regards initial credits in the wallet. We assume that the initial credit is zero. So new authors (for example young graduate students), or authors who depleted their wallets via too aggressive bidding (and therefore got a lower number of citations than predicted by themselves in the auction) would be cut off from even attempting to get the papers published. To avoid that, a certain fraction of papers should be reviewed in a traditional way but with much higher acceptance criteria. Alternatively, "sponsorship" could be applied, such as when the authors look for credit, their colleagues may decide to trust them and consequently grant the credit of some citations from their personal CWs (but if they do not value the paper highly, they consequently would not grant any credits). Hence, a researcher may want invest part of her $\mathrm{CW}$ in a paper by a promising young talent for a "profit" of future citations, if she judges the papers strong.

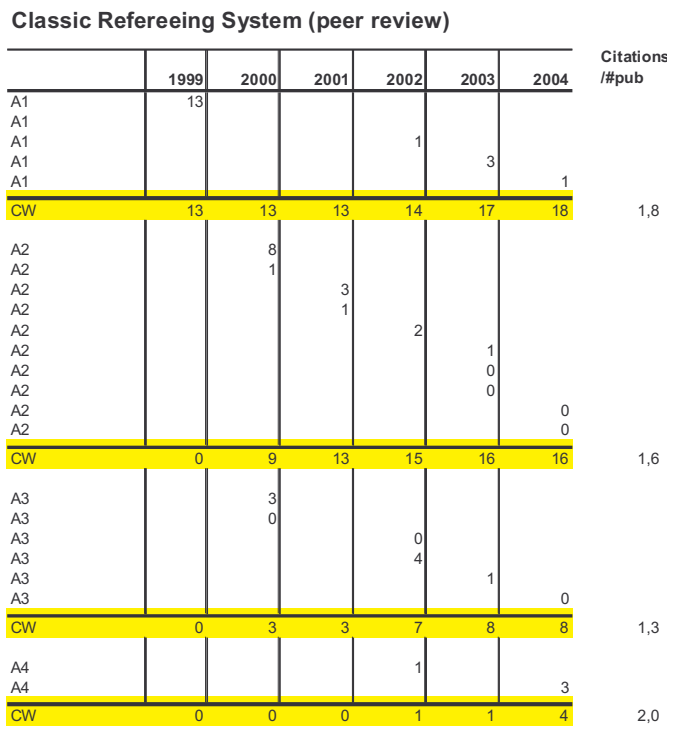

Table 3. The citations by May 2006 of 4 authors in Lecture Notes (AI \& CS) during 6 years (1999-2004)

With these explanations, let us consider another hypothetical example. In Table 3 there is the result of looking up the publications in LNCS, LNAI and their citations of 4 researchers for the period of 19992004. Again, one can identify several rankings. The first one is the absolute citations ranking, which is clearly led by A1 with 18 citations, followed by A2 with 16 citations, and by distant A3 with 8 , and ended by $\mathrm{A} 4$ with 4 . Using the ratio of citations to the number of publications, as described in [2], yields another order: A4 is the leader with 2 citations per publication, followed by A1 with 1.8, then A2 with 1.6, and finally A3 with 1.3

Another experiment, Table 4, represents a possible alternative scenario in the same period of time with a simulation of four auction behavior patterns (aggressive, cautious, very cautious, no-risk) of the same researchers. The blue squares mark the row of researchers that did not win the auction of the corresponding column. The column "Auction" indicates the number of citations that a researcher $A_{i}$ expects from his contributions, the column "W" indicates the accepted number of citations for every publication after the auction, and the following column "year" shows the number of citations that as of May 2006 the contributions have gathered from the publication. Here, for the sake of simplicity, the method for auction is the FPSB, where every author submits in a closed envelop the bid, defining how many citations the author offers to a conference or journal. This bid supposes to be lower than the amount of citations that the author will collect in the future, after the publication of his paper. 
Again several rankings can be discerned. The first one is the citations wallet (CW) ranking, as the number of remaining citations, after the auction. In the discussed case, this ranking is clearly led by $\mathrm{A} 2$ with 9 citations, followed by A1 with 7 citations, then A3 with 5, and ended by A4 with 4 . Note that the $\mathrm{CW}$ values are lower than the absolute number of citations received. However, the advantage is that $\mathrm{CW}$ reflects the additional number of citations, above the number of the expected citations, that the entire set of publications have generated for the author $A_{i}$ in a given time period. By regarding the ratio of citations per publication, we define a new content of citation wallet, which is the cumulative number of will be losing many auctions. Conversely, an author can increase his CW quickly by submitting highly cited papers, therefore making it easy for him to publish in the future.

\section{Conclusions and future work}

This work has introduced thoughts about the current peer refereeing procedure, about its drawbacks, and has suggested a new approach to selecting the papers for competitive conferences and journals, the citation auction which possesses interesting properties such as: reduced refereeing

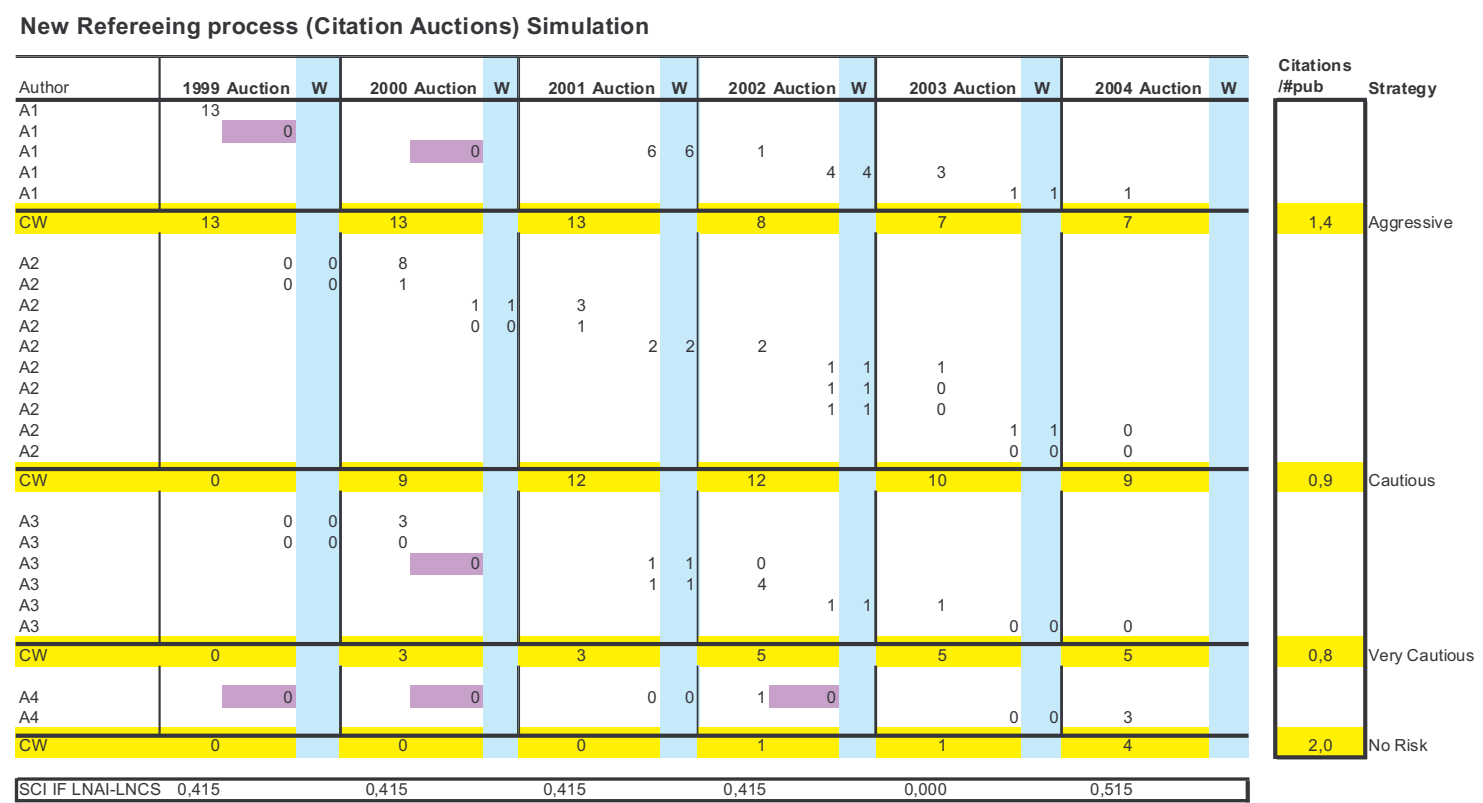

Table 4. Simulation of 4 strategies citation auctions, (blue squares denote unsuccessful bids)

citations per publication. This new CW content gives another order: led by A4 with $2 \mathrm{CW}$ per publication, followed by A1 with 1.8, then A2 with 0.9 and finally A3 with 0.8. In this case those ratios have not changed from Table 3.

The difference between tables 3 and 4 is that Table 3 shows the outcome of publishing the paper, in terms of citations while Table 4 shows the outcome compared to expectations. The former gives a more accurate measure of the quality of a paper, so the authors have an incentive to attempt to gain the maximum number of citations, at least the same number as the number of citations invested in the bid. Moreover, this citations auction system is selfregulating: if an author is repeatedly underperforming in citations (that is his contributions receive a lower number of citations than those invested) then his $\mathrm{CW}$ will eventually drop close to zero and this author will have difficulties in the future to assure the publication of his contributions in conferences or journals as he overload, higher motivation for authors to actively participate and explain their work well, more accurate fitting of papers and publications, and broader participation in conferences and post-refereeing. Its main drawback is the fact that the citation system is still inaccurate, for example how to distinguish the positive from the negative (critical) citations? There are also the following, new uncertainties, as for example the inflation and deflation of this new economic model.

The citation auction is defined in this paper together with its operation, and very preliminary illustrative examples are given with the analysis of the contributions to two publications: Proceedings of the Workshop on Parallel and Distributed Simulations (published by the IEEE Computer Science Press) and the Lecture Notes on Computer Science and Lecture Notes on Artificial Intelligence (published by Springer). In both cases four authors were analyzed 
over the years 2003-2005 in the first case and 19992004 in the second case. Next steps are:

1. Create a proof-of-concept of the citations auction review process with a workshop.

2. Explore what are the most appropriate auctions mechanisms.

3. Develop the technology for the bank of citations. This will use standard citation engines.

4. Study how to stabilize the resulting economic model based on citations. Perhaps some sort of "taxes" would be needed.

5. Obtain deeper knowledge of small scientific communities to handle them and make them cooperate with other communities more easily. Again "currencies" and taxes may help.

Finally, one can claim it is too late to adopt our approach as current publications' functioning has been adopted by everybody. The process of adopting Citation Auctions may last decades, but the economic background idea for peer-review will introduce efficiency in the scientific activities. And as former economic systems in the past were finally adopted, thus we expect citation auctions will be adopted too.

\section{Acknowledgements}

This work was supported in part by the Grant EU project $\mathrm{N}^{\circ} 34744$ ONE: Open Negotiation Environment, FP62005-IST-5, ICT-for Networked Businesses, and by grant OISE-0334667 from the National Science Foundation of US. The content of this paper does not necessarily reflect the position or policy of the sponsors; no official endorsement should be inferred or implied.

\section{References}

[1] Philip G. Altbach (2006) is director of the Center for International Higher Education, at Boston College. http://insidehighered.com/views/2006/05/08/altbach

[2] de la Rosa, J.Ll. (2005), Strategic R+D Plan of the University of Girona, 2005-2007, http://pserv.udg.es/dnn3/Default.aspx?alias=pserv.udg .es/dnn3/per

[3] Capen, Edward, Robert Clapp, and William Campbell. (1971). Competitive Bidding in High Risk Situations, Journal of Petroleum Technology, 23, 641-653.

[4] Lee, Juong-Sik, and Boleslaw K. Szymanski (2005). A Novel Auction Mechanism for Selling Time-Sensitive E-Services, $7^{\text {th }}$ International IEEE Conference on ECommerce Technology (CEC'05), Munich, Germany, pp. $75-82$.

[5] Lee, Juong-Sik, and Boleslaw K. Szymanski (2006). Auctions as a Dynamic Pricing Mechanism for eServices, Service Enterprise Integration, 131-156, Cheng Hsu (ed.), Kluwer, New York.
[6] Herschlag, Miriam, and Rami Zwick. (2000). Internet Auctions-A Popular and Professional Literature Review, Quarterly Journal of e-Commerce, 1(2), 161-186.

[7] Klemperer, Paul. (1999). Auction Theory: A Guide to the Literature, Journal of Economic Surveys, 13(3), 227- 260. Klemperer, Paul (ed.) (2000). The Economic Theory of Auctions, Volumes 1 \& 2, Cheltenham, UK: Edward Elgar. Laffont.

[8] McAfee, R., Preston, and John McMillan. (1987). Auctions and Bidding, Journal of Economic Literature, 25 (June), 699-738.

[9] Milgrom, Paul, R., and Robert J. Weber. (1982). A Theory of Auctions and Competitive Bidding, Econometrica, 50(5), 1089-1122.

[10] S. Mizzaro (2003). Quality Control in Scholarly Publishing: A New Proposal, Journal of the American Society for Information Science and Technology, 54(11):989-1005

[11] J. L. de la Rosa (2006). Outline of Citation Auctions, Frontiers in Artificial Intelligence and Applications AI Research \& Development, Vol. 146 (October), 299-307, IOS Press ISSN 0922-6389

[12] S. Mizzaro (2000). An automatically refereed scholarly electronic journal. In C. Bavec, M. Gams, V. Rajkovic, I. Rozman, J. Gyorkos, editors, Information Society 2000 - Proceedings of the Third International Conference, pages 121-126, Ljubljana, Slovenia, 17-19 October 2000. ISBN: 961 6303-26-0.

[13] S. Mizzaro (1999). A concrete proposal for an automatically refereed scholarly electronic journal. Research Report UDMI/07/99, Department of Mathematics and Computer Science, University of Udine, April 1999. Presented at a Workshop at ECDL'99 Conference, Paris.

[14] A. Pesenhofer, R. Mayer, A. Rauber (2006). Improving Scientific Conferences by enhancing Conference Management Systems with Information Mining Capabilities, Proceedings IEEE International Conference on Digital Information Management (ICDIM 2006), IEEE, p. $359-366$

[15] J. E. Hirsch (2005). An index to quantify an individual's scientific research output, The National Academy of Sciences of the USA, PNAS 2005; 102; 16569-16572; published online Nov 7, 2005; doi:10.1073/pnas.0507655102, updated at Nov 2006.

[16] C. Carrillo, J. L. de la Rosa, A. Moreno, E. Muntaner, S. Delfin and A. Canals (2006) Social Currencies and Knowledge Currencies, Frontiers in Artificial Intelligence and Applications - AI Research \& Development ISSN 0922-6389, Vol. 146 pp. 266-274, October 2006, IOS Press, Amsterdam, The Netherlands

[17] David A. Vise (2005). The Google Story, Pan Mac Millan Ed. 